\title{
Applicability of the parallel-clock model to duration discrimination
}

\author{
HANNES EISLER \\ University of Stockholm, S-113 85 Stockholm, Sweden
}

\begin{abstract}
The parallel-clock model assumes that when an observer is presented with two durations in succession, the total subjective duration (corresponding to the sum of first and second durations) and the second duration are each accumulated in a separate sensory register. In dealing with some relation between the two durations, the observer compares the difference between the contents of the two registers with the contents of the second register. With the further assumption that the psychophysical power law is valid for the continuum of time, the model has previously been shown to account well for duration scaling data. After adapting the model in the vein of Thurstone for duration discrimination data, it was tried out on such data gathered by Allan (1977) from 13 observers. With chi-square as the examined statistic, (1) five distributions were compared and the normal one chosen, (2) one categorization model, three choice models, and two linear regression models were compared with the 3-parameter version of the parallel-clock model, which proved superior, and (3) a 4-5-parameter version of the parallel-clock model, assuming a discontinuity in the psychophysical function, was shown to yield an excellent fit in terms of the Kolmogorov-Smirnov statistic.
\end{abstract}

The parallel-clock (PC) model (Eisler, 1975) describes the behavior of observers who are presented with two successive durations and required to deal with some relation between them, for example, to make the second duration as long as the first (reproduction of time intervals). Unlike the classic interpretation, which envisages that the first duration is stored in memory and that this memory is compared with the second, the PC model assumes two sensory registers. One of these accumulates not just the first, but the total subjective duration (from the onset of the first physical duration to the of fset of the second $^{1}$ ), while the other accumulates the second subjective duration. Thus, during the second (physical) duration, two registers ("clocks") are running simultaneously-hence the name "parallel-clock model." The distinction between physical duration (in seconds) and subjective duration is of paramount importance. If there were no difference, that is, if time perception were veridical (subjective duration proportional to physical duration), the PC model would be void, since it would be data-equivalent to the classic model. The quantities an observer deals with in comparing the two durations-whatever the experimental taskare the difference in content between the two registers, and the contents of the second register, respectively. The model is illustrated in Figure 1. The middle pair of (equally long) double-pointed arrows

This investigation was supported by the Swedish Council for Research in the Humanities and Social Sciences. I am indebted to $O$. Guilbaud for statistical assistance. Requests for reprints should be sent to Hannes Eisler, Department of Psychology, University of Stockholm, Box 6706, S-113 85 Stockholm, Sweden. indicates the point in time when the two successive durations are experienced as equally long; for the left pair of arrows, the first duration is experienced as longer, and for the right pair, it is the second.

The model was devised with ratio-setting tasks, particularly duration reproduction, in mind. Denot-

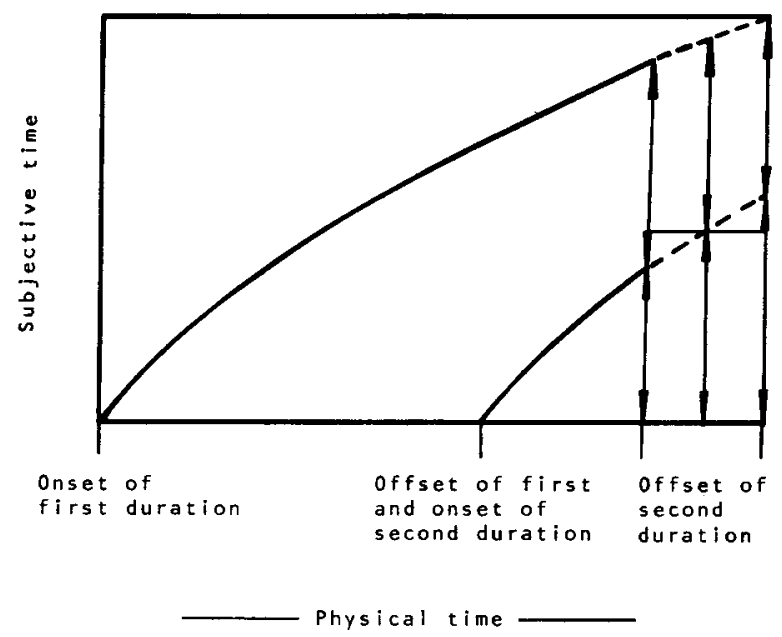

Figure 1. Subjective duration, $\psi$, as a function of physical duration, $\phi$, according to the parallel-clock model. The upper curve indicates the accumulation of the total duration (first + second). Note that it does not terminate at the offset of the first duration. The lower curve indicates the accumulation of the second duration. The two-pointed arrows show the compared quantities, the difference between total and second subjective duration, and second subjective duration, respectively. For the middle pair of arrows, first and second duration are experienced as equal; for the left, the first, and for the right, the second duration is experienced as longer. 
ing subjective duration, that is, the contents of the registers, by $\psi$, the upper curve in Figure 1 shows $\psi_{\text {tot }}$, the subjective duration from the start of the first time interval, and the lower curve, $\psi_{2}$, the subjective duration of the second time interval. At the point in time at which the two durations are experienced as equal, we have $\psi_{\text {tot }}-\psi_{2}=\psi_{2}$ : the two double-pointed arrows in Figure 1 are equally long. As the psychophysical function, relating subjective to physical duration, Stevens' power function of the form

$$
\psi(\phi)=\alpha\left(\phi-\phi_{0}\right)^{\beta}
$$

was derived, where $\psi$ denotes subjective duration and $\phi$ physical duration, $\alpha$ is the scale unit, $\phi_{0}$ the subjective zero, and $\beta$ the exponent. The PC model accounted well for the data obtained in a series of experiments (Eisler, 1975). Its generality was shown by applying it successfully to a great number of scaling $^{2}$ experiments, yielding about 500 exponents scattering around .9 (Eisler, 1976).

The first paper (Eisler, 1975) emphasized duration scaling, but it also pointed out that the PC model could explain some findings in duration discrimination experiments that are exclusive for the continuum of time. However, no quantitative analysis of discrimination data was carried out. In 1977, Allan published a duration discrimination study. Her data were reanalyzed by Jamieson (1977), who tried out a number of models. The aim of the present study was to adapt the PC model for duration discrimination and to try it out on Allan's data.

Three analyses are carried out: (1) comparison for the stochastic version of the PC model between five distribution functions; (2) comparison of the PC model with six other models; and (3) examination of the goodness of fit of an improved version of the PC model in an absolute sense.

\section{THE PC MODEL FOR DISCRIMINATION}

Incorporating the power law, Equation 1, into the PC model for duration reproduction, requiring the second duration to match the first one, yields

$$
\alpha\left(\phi_{1}+\phi_{2}-\phi_{0}\right)^{\beta}-\alpha\left(\phi_{2}-\phi_{0}\right)^{\beta}=\alpha\left(\phi_{2}-\phi_{0}\right)^{\beta},
$$

that is,

$$
\left.\phi_{1}+\phi_{2}-\phi_{0}\right)^{\beta}-2\left(\phi_{2}-\phi_{0}\right)^{\beta}=0,
$$

where $\phi_{1}$ and $\phi_{2}$ are the first and second physical duration, respectively, and the $\alpha$ s cancel and are set $=1$. Equation 2 says that the difference between the total and the second subjective duration equals the second subjective duration, in accordance with the PC model (see Figure 1).
For discrimination experiments, the deterministic version of Equation 3 has to be replaced by a stochastic one, suitably in the vein of Thurstone. The probability, P, of a "first longer" judgment is given in the following way:

$$
\left(\phi_{1}+\phi_{2}-\phi_{0}\right)^{\beta}-2\left(\phi_{2}-\phi_{0}\right)^{\beta}=s \zeta,
$$

where $P=F(\xi)$ and $F$ is a distribution function of a symmetrically distributed variable, with mean 0 and variance 1 , and $s$ is the comparatal dispersion or possibly just a scale unit. $\zeta$ is used rather than $z$ because, so far, no decision has been made about the form of distribution; $z$ is used if $F$ refers to the normal distribution.

\section{DATA TREATMENT}

\section{Allan's (1977) Experiments}

Thirteen observers were required to indicate, by pressing the appropriate button, which of two sequentially presented visual stimuli was the longer. Two sets of durations were used, viz., $\{.9,1.0,1.1$, $1.2\}$ and $\{.07, .10, .13, .16\}$ sec. Eight observers were presented with pairs drawn from the first set, and 9 were presented with pairs drawn from the second; thus, 4 of the 13 observers participated in both partexperiments. (Allan's Experiment 2 consisted of the second runs of these four observers.) From each of the two stimulus sets, all pairs, including equal stimuli (but excluding pairs made up of stimuli more than one step apart), were presented in random order, that is, 10 different pairs for each set. For the first set, the pairs were: $.9, .9 ; 1.0, .9 ; .9,1.0 ; 1.0,1.0$; $1.1,1.0 ; 1.0,1.1 ; 1.1,1.1 ; 1.2,1.1 ; 1.1,1.2 ; 1.2,1.2$. For the second set, they were similar. Table 1 gives the total number of presentations of each pair, together with the number of "first longer" judgments. ${ }^{3}$ These data are used in the forthcoming computations.

\section{General Remarks Regarding the Computations}

All computations consisted of minimizing, by means of Chandler's (1969) routine STEPIT,

$$
\begin{aligned}
\chi^{2}=\sum_{\mathrm{i}=1}^{10}\left[\frac{\left(\mathrm{O}_{1 \mathrm{i}}-\mathrm{P}_{1 \mathrm{i}}\right)^{2}}{\mathrm{P}_{1 \mathrm{i}}}\right. & \left.+\frac{\left(\mathrm{O}_{2 \mathrm{i}}-\mathrm{P}_{2 \mathrm{i}}\right)^{2}}{\mathrm{P}_{2 \mathrm{i}}}\right] \\
& =\sum_{\mathrm{i}=1}^{10} \frac{\left(\mathrm{O}_{1 \mathrm{i}}-\mathrm{P}_{1 \mathrm{i}}\right)^{2} \mathrm{~N}_{\mathrm{i}}}{\mathrm{P}_{1 \mathrm{i}}\left(\mathrm{N}_{\mathrm{i}}-\mathrm{P}_{1 \mathrm{i}}\right)}
\end{aligned}
$$

where $O$ denotes empirical (observed) and $P$, predicted frequencies. Subscripts 1 and 2 refer to frequencies of "first longer" and "second longer" judgments, and $\mathrm{N}_{\mathrm{i}}$ refers to the number of presentations of a pair. The predicted frequencies are obtained by multiplying the predicted proportions with 
Table 1

Frequency of First Duration Judged Longer (D) and Total Frequency of Presentation of a Pair (P) for Each Subject in Allan's (1977) Experiments 1 and 2

\begin{tabular}{|c|c|c|c|c|c|c|c|c|c|c|c|c|c|c|c|c|c|c|c|c|}
\hline \multirow{3}{*}{$\begin{array}{l}\text { Sub- } \\
\text { ject }\end{array}$} & \multicolumn{20}{|c|}{ First Stimulus/Second Stimulus } \\
\hline & \multicolumn{2}{|c|}{$.90 / .90$} & \multicolumn{2}{|c|}{$1.00 / .90$} & \multicolumn{2}{|c|}{$.90 / 1.00$} & \multicolumn{2}{|c|}{$1.00 / 1.00$} & \multicolumn{2}{|c|}{$1.10 / 1.00$} & \multicolumn{2}{|c|}{$1.00 / 1.10$} & \multicolumn{2}{|c|}{$1.10 / 1.10$} & \multicolumn{2}{|c|}{$1.20 / 1.10$} & \multicolumn{2}{|c|}{$1.10 / 1.20$} & \multicolumn{2}{|c|}{$1.20 / 1.20$} \\
\hline & D & $\mathbf{P}$ & D & $\mathbf{P}$ & $\mathrm{D}$ & $\mathbf{P}$ & D & $\mathbf{P}$ & D & $\mathbf{P}$ & D & $\mathbf{P}$ & $\mathrm{D}$ & $\mathbf{P}$ & D & $\mathbf{P}$ & D & $\mathbf{P}$ & D & $\mathbf{P}$ \\
\hline $\begin{array}{l}\text { L.P. } \\
\text { S.S. } \\
\text { B.J. } \\
\text { K.F. } \\
\text { S.M. } \\
\text { J.B. } \\
\text { J.A. } \\
\text { L.S. }\end{array}$ & $\begin{array}{r}99 \\
113 \\
41 \\
54 \\
106 \\
63 \\
69 \\
67\end{array}$ & $\begin{array}{l}148 \\
150 \\
115 \\
150 \\
174 \\
120 \\
109 \\
119\end{array}$ & $\begin{array}{r}103 \\
129 \\
65 \\
66 \\
134 \\
98 \\
81 \\
80\end{array}$ & $\begin{array}{l}150 \\
150 \\
119 \\
150 \\
176 \\
119 \\
110 \\
117\end{array}$ & $\begin{array}{r}45 \\
73 \\
13 \\
13 \\
111 \\
19 \\
29 \\
58\end{array}$ & $\begin{array}{l}149 \\
149 \\
117 \\
150 \\
177 \\
120 \\
110 \\
116\end{array}$ & $\begin{array}{r}50 \\
108 \\
24 \\
32 \\
121 \\
53 \\
45 \\
60\end{array}$ & $\begin{array}{l}150 \\
150 \\
118 \\
150 \\
176 \\
119 \\
110 \\
118\end{array}$ & $\begin{array}{r}73 \\
127 \\
48 \\
35 \\
135 \\
75 \\
74 \\
59\end{array}$ & $\begin{array}{l}150 \\
150 \\
119 \\
149 \\
177 \\
120 \\
109 \\
116\end{array}$ & $\begin{array}{l}17 \\
86 \\
13 \\
16 \\
94 \\
22 \\
27 \\
36\end{array}$ & $\begin{array}{l}150 \\
150 \\
118 \\
149 \\
174 \\
120 \\
110 \\
114\end{array}$ & $\begin{array}{r}42 \\
109 \\
14 \\
24 \\
111 \\
40 \\
42 \\
38\end{array}$ & $\begin{array}{l}150 \\
148 \\
118 \\
119 \\
173 \\
119 \\
110 \\
115\end{array}$ & $\begin{array}{r}66 \\
131 \\
42 \\
37 \\
112 \\
60 \\
65 \\
48\end{array}$ & $\begin{array}{l}149 \\
149 \\
112 \\
149 \\
178 \\
118 \\
109 \\
117\end{array}$ & $\begin{array}{r}25 \\
92 \\
1 \\
11 \\
94 \\
11 \\
17 \\
21\end{array}$ & $\begin{array}{l}150 \\
149 \\
118 \\
150 \\
176 \\
120 \\
109 \\
118\end{array}$ & $\begin{array}{r}42 \\
118 \\
28 \\
27 \\
100 \\
25 \\
32 \\
32\end{array}$ & $\begin{array}{l}149 \\
149 \\
116 \\
150 \\
173 \\
120 \\
110 \\
117\end{array}$ \\
\hline \multirow{3}{*}{$\begin{array}{l}\text { Sub- } \\
\text { ject }\end{array}$} & \multicolumn{20}{|c|}{ First Stimulus/Second Stimulus } \\
\hline & \multicolumn{2}{|c|}{$.07 / .07$} & \multicolumn{2}{|c|}{$.10 / .07$} & \multicolumn{2}{|c|}{$.07 / .10$} & \multicolumn{2}{|c|}{$.10 / .10$} & \multicolumn{2}{|c|}{$.13 / .10$} & \multicolumn{2}{|c|}{$.10 / .13$} & \multicolumn{2}{|c|}{$.13 / .13$} & \multicolumn{2}{|c|}{$.16 / .13$} & \multicolumn{2}{|c|}{$.13 / .16$} & \multicolumn{2}{|c|}{$.16 / .16$} \\
\hline & $\mathrm{D}$ & $\mathbf{P}$ & D & $\mathbf{P}$ & D & $\mathbf{P}$ & D & $\mathbf{P}$ & $\mathrm{D}$ & $\mathbf{P}$ & D & $\mathbf{P}$ & D & $\mathbf{P}$ & D & $\mathbf{P}$ & D & $\mathbf{P}$ & D & $\mathbf{P}$ \\
\hline $\begin{array}{l}\text { M.G. } \\
\text { L.S. } \\
\text { P.B. } \\
\text { S.A. } \\
\text { D.O. } \\
\text { D.E. } \\
\text { B.J. } \\
\text { J.B. } \\
\text { J.A. }\end{array}$ & $\begin{array}{r}147 \\
185 \\
187 \\
65 \\
97 \\
59 \\
81 \\
69 \\
87\end{array}$ & $\begin{array}{l}221 \\
235 \\
239 \\
106 \\
148 \\
103 \\
149 \\
179 \\
120\end{array}$ & $\begin{array}{r}208 \\
215 \\
231 \\
89 \\
137 \\
93 \\
98 \\
168 \\
111\end{array}$ & $\begin{array}{l}224 \\
235 \\
239 \\
109 \\
148 \\
106 \\
148 \\
180 \\
120\end{array}$ & $\begin{array}{r}32 \\
75 \\
99 \\
25 \\
39 \\
51 \\
34 \\
8 \\
26\end{array}$ & $\begin{array}{l}229 \\
235 \\
238 \\
108 \\
149 \\
104 \\
148 \\
180 \\
119\end{array}$ & $\begin{array}{r}134 \\
142 \\
183 \\
53 \\
86 \\
63 \\
48 \\
69 \\
79\end{array}$ & $\begin{array}{l}219 \\
239 \\
238 \\
110 \\
149 \\
107 \\
145 \\
179 \\
120\end{array}$ & $\begin{array}{r}207 \\
194 \\
227 \\
73 \\
131 \\
81 \\
78 \\
162 \\
105\end{array}$ & $\begin{array}{l}226 \\
235 \\
238 \\
110 \\
150 \\
114 \\
146 \\
180 \\
120\end{array}$ & $\begin{array}{r}51 \\
58 \\
72 \\
19 \\
19 \\
44 \\
24 \\
9 \\
15\end{array}$ & $\begin{array}{l}258 \\
236 \\
235 \\
107 \\
149 \\
108 \\
147 \\
178 \\
120\end{array}$ & $\begin{array}{r}112 \\
106 \\
124 \\
42 \\
61 \\
50 \\
42 \\
60 \\
54\end{array}$ & $\begin{array}{l}218 \\
235 \\
235 \\
110 \\
147 \\
102 \\
147 \\
180 \\
120\end{array}$ & $\begin{array}{r}191 \\
140 \\
203 \\
60 \\
112 \\
58 \\
66 \\
120 \\
79\end{array}$ & $\begin{array}{l}225 \\
236 \\
240 \\
110 \\
149 \\
108 \\
149 \\
180 \\
120\end{array}$ & $\begin{array}{l}19 \\
29 \\
29 \\
30 \\
34 \\
41 \\
30 \\
13 \\
13\end{array}$ & $\begin{array}{l}223 \\
239 \\
238 \\
107 \\
150 \\
107 \\
149 \\
180 \\
120\end{array}$ & $\begin{array}{r}94 \\
70 \\
106 \\
31 \\
64 \\
27 \\
38 \\
43 \\
35\end{array}$ & $\begin{array}{l}225 \\
238 \\
239 \\
109 \\
147 \\
104 \\
147 \\
180 \\
120\end{array}$ \\
\hline
\end{tabular}

$\mathrm{N}_{\mathrm{i}}$, and the predicted proportions are obtained from, for example, Equation 4 by estimating $\phi_{0}, \beta$, and $s$, computing $\zeta$, and finding the proportion corresponding to $\zeta$ from the pertinent distribution function.

If no parameter were estimated, the number of degrees of freedom would be 10 , since all 10 pairs are presented independently. (Jamieson, 1977, incorrectly assumes 9 .) The number of degrees of freedom of the chi-square distribution is thus 10 minus the number of free (fitted) parameters; for the PC model (Equation 4), the number of free parameters is 3 $\left(\phi_{0}, \beta\right.$, and $\left.s\right)$, and thus the number of degrees of freedom is 7 .

\section{Which Distribution?}

In the following, it is assumed that subjective duration $\psi$ may be a stochastic variable, although, strictly, Equation 1 denotes a deterministic variable.

Five distributions were tried out.

(1) The rectangular distribution. There are two rationales: (a) The inertia of the "comparator"' (where the difference between the contents of the two sensory registers is compared with the contents of the second register) may follow a rectangular distribution; or (b) the difference in the left term of Equation 4 may be described by a quantal psychometric function (see, e.g., Guilford, 1954, pp. 142-144).

(2) The triangular distribution. Again, there are two alternatives: (a) If the two subjective durations in Equation 4 are independent and each one is distributed rectangularly (quantal psychometric functions), the difference is distributed triangularly; or (b) analogous to Kristofferson's (e.g., 1977) assumption that a triangular distribution generates the psychophysical function for duration, a triangular distribution may hold for the difference of the two subjective durations.

(3) Distribution for the difference of two independent triangularly distributed stochastic variables. ${ }^{4}$ We assume a triangular distribution (see again, Kristofferson, 1977) for each one of the two terms in the left-hand side of Equation 4.

(4) The logistic. It is suggested by, for example, Luce (1959).

(5) The normal distribution in accordance with Thurstone's model for pair comparisons (see, e.g., Guilford, 1954, chap. 7).

The results in terms of chi-square values are given in Table 2. There is no great difference between the distributions except for the rectangular one, although the logistic seems best. However, computations with an improved version of the PC model show that the normal distribution is somewhat better than the triangular, the difference of triangular, and the logistic distributions. Therefore, the normal distribution is used in the sequel. Obviously, the form of the distribution, except for the first one, is not crucial for the PC model. 
Table 2

Chi-Square Values for Five Distributions for Each Subject in Allan's (1977) Experiments 1 and 2 for the Parallel-Clock Model

\begin{tabular}{|c|c|c|c|c|c|}
\hline \multirow[b]{2}{*}{ Subject } & \multicolumn{5}{|c|}{ Distribution } \\
\hline & Rectangular & Triangular & $\begin{array}{c}\text { Difference of } \\
\text { Triangular }\end{array}$ & Logistic & Normal \\
\hline & \multicolumn{5}{|c|}{ Long Durations } \\
\hline L.P. & 32.1 & 27.2 & 27.9 & 27.1 & 27.8 \\
\hline S.S. & 7.9 & 4.8 & 5.2 & 4.5 & 4.9 \\
\hline B.J. & 20.9 & 21.1 & 21.8 & 22.2 & 21.8 \\
\hline K.F. & 19.6 & 17.8 & 17.9 & 17.8 & 17.8 \\
\hline S.M. & 11.9 & 12.2 & 12.1 & 12.2 & 12.2 \\
\hline J.B. & 12.6 & 8.1 & 7.8 & 7.5 & 7.8 \\
\hline J.A. & 6.4 & 4.4 & 5.0 & 4.7 & 4.9 \\
\hline \multirow[t]{2}{*}{ L.S. } & 7.2 & 9.0 & 8.3 & 8.6 & 8.3 \\
\hline & \multicolumn{5}{|c|}{ Short Durations } \\
\hline M.G. & 24.1 & 12.7 & 12.8 & 12.0 & 12.5 \\
\hline L.S. & 21.5 & 8.5 & 7.7 & 7.2 & 7.4 \\
\hline P.B. & 71.0 & 30.7 & 27.9 & 29.5 & 27.8 \\
\hline S.A. & 15.7 & 12.2 & 12.7 & 11.9 & 12.5 \\
\hline D.O. & 16.7 & 16.4 & 16.4 & 17.0 & 16.5 \\
\hline D.E. & 24.4 & 26.1 & 25.8 & 26.2 & 25.9 \\
\hline B.J. & 11.8 & 7.5 & 8.1 & 7.2 & 7.9 \\
\hline J.B. & 71.6 & 39.6 & 36.3 & 32.0 & 34.6 \\
\hline J.A. & 20.0 & 10.2 & 9.8 & 9.2 & 9.5 \\
\hline Sum & 395 & 268 & 263 & 257 & 260 \\
\hline
\end{tabular}

\section{Model Comparisons}

In her paper, Allan (1977) suggested a categorization model to account for her data, but did not undertake a thorough quantitative analysis. Jamieson (1977) analyzed her data, with chi-square as the criterion of fit, both for her model and for three versions of Luce's (1959; Luce \& Galanter, 1963) choice model. Here, the model comparisons include three more models, viz., two linear regression models in the vein of Hellström (1977) and the PC model.

The use of subscripts in the following sections may require some explanation. The general subscript " $i$ " denotes a value connected with the particular stimulus (duration). For example, $\psi_{\mathrm{i}}$ in the choice models takes four different values of subjective duration, corresponding to the four stimuli. The digit subscripts " 1 " and " 2 " used in the formulas denote the "first" and "second" stimulus of a pair. Thus, in Equation 7, for example, $\psi_{1}$ may represent any of the four durations, and $\psi_{2}$ may stand for the next shorter, the same, or the next longer physical duration.

(1) Allan's categorization model. According to this model, each duration is categorized, independently of the other stimulus in the pair, as "long" with a certain probability, $p_{i}$, where subscript $i$ refers to duration i. (The probability of the same stimulus's being categorized as "short" is, of course, 1- $p_{i}$.) If the two stimuli are categorized differently, the response is in accordance with that outcome; if both stimuli are categorized as "short," the probability for the response "first longer" is q; and if they are both categorized as "long," the corresponding prob- ability is $r$. Thus, probability $P$ for obtaining a "first longer" response is

$$
P=p_{1}\left(1-p_{2}\right)+\left(1-p_{1}\right)\left(1-p_{2}\right) q+p_{1} p_{2} r
$$

where $p_{1}$ and $p_{2}$ are the probabilities of categorizing the first and second diration, respectively, as "long." This model has five free parameters, although in the computations six are used: the $p$ values for the four durations, and $q$ and $r$ (cf. Jamieson, 1977).

All three choice model versions are elaborations of the simple choice model:

$$
\mathrm{P}=\frac{\psi_{1}}{\psi_{1}+\psi_{2}}
$$

where $\psi_{i}$ is the subjective duration of stimulus $i$.

(2) The assimilation model replaces $\psi_{1}$ with a weighted average of $\psi_{1}$ and the adaptation level, defined as

$$
\mathbf{A}=\left(\prod_{i=1}^{4} \psi_{i}\right)^{1 / 4}
$$

The probability of a "first longer" judgment becomes

$$
P=\frac{q \psi_{1}+(1-q) A}{q \psi_{1}+(1-q) A+\psi_{2}}
$$

where $\psi_{1}$ and $\psi_{2}$ are the subjective durations of the first and second stimuli in the pair, and $q$ and $1-q$, 
the weights. This model has four free parameters, three subjective durations (the lowest is fixed arbitrarily at unity), and q.

(3) The bias model introduces a response bias $\eta$ in Equation 7, yielding

$$
\mathrm{P}=\frac{\psi_{1}}{\psi_{1}+\eta \psi_{2}}
$$

Like the previous model, there are four free parameters, the three subjective durations, and $\eta$.

(4) The assimilation + bias model combines the two previous models:

$$
P=\frac{q \psi_{1}+(1-q) A}{q \psi_{1}+(1-q) A+\eta \psi_{2}},
$$

and accordingly features five free parameters, the three subjective durations and $q$ and $\eta$.

The linear regression models are built on the quantity

$$
y=q \phi_{1}{ }^{\beta}-r \phi_{2}{ }^{\beta}+C,
$$

where $q$ and $r$ are weights, reflecting the attention paid to the first and second subjective durations, respectively, and $C$ can be considered as composed of adaptation levels weighted differently and interacting with the two subjective durations. The number of free parameters is four: $q, r, C$, and $\beta$.
(5) The logistic linear regression model uses the logistic as distribution. We obtain

$$
\mathrm{P}=\frac{1}{1+\exp (-\mathrm{y})} \text {. }
$$

(6) The normal linear regression model assumes $\mathrm{P}=\Phi(\mathrm{y})$, where $\Phi$ is the normal distribution function.

(7) The parallel-clock model (Equation 4), with $P=\Phi(\mathrm{z})$, has three free parameters.

Table 3 gives the chi-square values for these seven models. ${ }^{5}$ When making comparisons, one has to consider the number of degrees of freedom, which are 5 , $6,6,5,6,6$, and 7 , in that order. Since the majority of fits have to be rejected for all models, the number of "good fits" ( $p>.3$ ) is counted instead. According to this criterion, the $\mathrm{PC}$ model proves superior, with 6 good fits out of 17 . However, to be best is not enough. The result is, of course, not satisfactory for the PC model either.

One may ask whether certain observers are fitted better by some model other than the PC. Particularly, Observers S.A. and B.J. (short durations) seem to be candidates for the assimilation + bias choice model. However, for S.A. the ratio between the longest and the shortest subjective durations (corresponding to .07 and $.16 \mathrm{sec}$ ) exceeds 33 , which is absurd. The same holds true for B.J., with a ratio exceeding 19. In that connection, it is noteworthy that the exponents of the power function obtained from the linear regression models are all reasonable,

\begin{tabular}{|c|c|c|c|c|c|c|c|}
\hline \multirow[b]{3}{*}{ Subject } & \multirow{3}{*}{$\begin{array}{l}\text { Categorization } \\
\text { Model (5) }\end{array}$} & \multirow{3}{*}{ Assimilation (4) } & \multicolumn{2}{|c|}{ Choice Models } & \multirow{2}{*}{\multicolumn{2}{|c|}{ Linear Regression Models }} & \multirow{3}{*}{$\begin{array}{c}\text { Parallel-Clock } \\
\text { Model (3) }\end{array}$} \\
\hline & & & & \multirow{2}{*}{$\begin{array}{l}\text { Assimilation } \\
+ \text { Bias (5) }\end{array}$} & & & \\
\hline & & & Bias (4) & & Logistic (4) & Normal (4) & \\
\hline & \multicolumn{7}{|c|}{ Long Durations } \\
\hline L.P. & 33.5 & 122.7 & 104.4 & 9.9 & 29.4 & 30.2 & 27.8 \\
\hline S.S. & $6.1 *$ & 320.6 & $5.3^{*}$ & $4.2^{*}$ & $4.5^{*}$ & $4.9 *$ & $4.9^{*}$ \\
\hline B.J. & 52.8 & 339.1 & 34.7 & 14.8 & 22.5 & 22.0 & 21.8 \\
\hline K.F. & 18.8 & 473.9 & 32.8 & 6.4 & 18.4 & 18.2 & 17.8 \\
\hline S.M. & 10.2 & 132.2 & 16.0 & 9.5 & 12.0 & 12.0 & 12.2 \\
\hline J.B. & 27.9 & 104.8 & 47.8 & 16.1 & 7.7 & 7.9 & $7.6^{*}$ \\
\hline J.A. & 17.4 & 39.5 & 37.1 & $4.4^{*}$ & $4.8^{*}$ & $5.0^{*}$ & $4.9^{*}$ \\
\hline \multirow[t]{2}{*}{ L.S. } & $4.3^{*}$ & 35.4 & 73.1 & 9.4 & $6.2^{*}$ & $6.0^{*}$ & $8.3^{*}$ \\
\hline & \multicolumn{7}{|c|}{ Short Durations } \\
\hline M.G. & 292.4 & 29.3 & 49.9 & 28.1 & 11.7 & 12.0 & 12.5 \\
\hline L.S. & 87.4 & 47.7 & 217.1 & 39.3 & 7.3 & $6.5 *$ & $7.4^{*}$ \\
\hline P.B. & 188.7 & 98.9 & 159.5 & 64.7 & 24.3 & 21.8 & 27.8 \\
\hline S.A. & 14.4 & 31.1 & 33.5 & $5.6^{*}$ & 11.4 & 12.0 & 12.5 \\
\hline D.O. & 104.5 & 9.2 & 38.2 & 8.9 & 18.0 & 17.5 & 16.5 \\
\hline D.E. & 22.4 & 32.8 & 63.1 & 31.5 & 24.7 & 24.4 & 25.9 \\
\hline B.J. & 17.2 & 140.0 & 42.6 & $1.6^{*}$ & 8.3 & 9.2 & $7.9^{*}$ \\
\hline J.B. & 265.1 & 119.4 & 19.8 & 15.3 & 25.5 & 27.5 & 34.6 \\
\hline J.A. & 103.8 & 39.0 & 85.9 & 29.5 & 8.4 & 8.6 & 9.5 \\
\hline Sum & 1267 & 2116 & 1061 & 299 & 245 & 246 & 260 \\
\hline Fits & 2 & 0 & 1 & 4 & 3 & 4 & 6 \\
\hline
\end{tabular}
both in terms of what has been obtained in scaling

Table 3

Chi-Square Values for Seven Models for Each Subject in Allan's (1977) Experiments 1 and 2

Note-The numbers in parentheses indicate the number of free parameters for each model. "Fits" indicates the number of good fits. ${ }^{*} p>.3$. 
experiments and in comparison with the results from the PC model: They vary between .47 and 1.01 . For the corresponding values obtained from the PC model, see Table 4.

\section{An Improved Version of the PC Model}

A striking feature of the data obtained in the duration scaling experiments (Eisler, 1975) was that all observers showed a break, or discontinuity, in the psychophysical function for at least one of the different tasks. ${ }^{6}$ This break could be described by different values for the subjective zero, $\phi_{0}$, or the scale unit, $\alpha$, or both, on the two sides of the break. Thus, Equation 1 is replaced by the following expressions:

$$
\left.\begin{array}{lll}
\psi_{\mathrm{br}}(\phi)=\alpha_{\mathrm{b}}\left(\phi-\phi_{0 \mathrm{~b}}\right)^{\beta} & \text { for } & \phi_{0 \mathrm{~b}}<\phi<\phi^{\prime} \\
\psi_{\mathrm{br}}(\phi)=\alpha_{\mathrm{a}}\left(\phi-\phi_{0 \mathrm{a}}\right)^{\beta} & \text { for } & \phi^{\prime}<\phi
\end{array}\right\},
$$

where $\phi^{\prime}$ is the physical duration value of the break. The accordingly generalized PC model for discrimination becomes:

$$
\begin{aligned}
\psi_{\mathrm{br}}\left(\phi_{1}+\phi_{2}\right)-2 \psi_{\mathrm{br}}\left(\phi_{2}\right) & =\alpha\left(\phi_{1}+\phi_{2}-\phi_{0}\right)^{\beta}-2 \alpha\left(\phi_{2}-\phi_{0}\right)^{\beta} \\
& =\mathrm{sz}
\end{aligned}
$$

with $\mathrm{z}$ being the normal deviate and the values of $\alpha$ and $\phi_{0}$ in each one of the two terms depending on the position of $\phi^{\prime}$ relative to $\phi_{1}+\phi_{2}$ and $\phi_{2}$. Look, for instance, at Observer B.J. (short durations) in Table 4 . His break lies at $.15 \mathrm{sec}$. That means that for the first pair, $.07+.07$ and .07 , both $<.15$, both $\phi_{0}$ values $=.033$. For the next pair, $.10+.07$ and .07 , $.17>.15$, whereas $.07<.15$. Thus, $\phi_{0}$ for the left term equals .040 , and for the right, .033 . This condition holds for all remaining pairs except the last two. For the next to last, $.13+.16$ and .16 , both $>.15$, both $\phi_{0}$ values equal .40 . The same is the case for the last pair, $.16+.16$ and .16 . The argument regarding the $\alpha$ values is similar.

The model of Equation 14 was tried out (1) allowing different values of the subjective zeros $\phi_{0}$, (2) allowing different values of the scale units, $\alpha$, one of which retains the value 1 , and (3) both. This was done for the data of all observers, placing the break at all 10 possible positions within the experimental range. Furthermore, another version was tried out, in which it was assumed that the parameter values were different for the middle two terms of Equation 14 throughout (no break assumed), that is,

$$
\alpha_{\mathrm{a}}\left(\phi_{1}+\phi_{2}-\phi_{0 \mathrm{a}}\right)^{\beta}-2 \alpha_{\mathrm{b}}\left(\phi_{2}-\phi_{0 \mathrm{~b}}\right)^{\beta}=\mathrm{sz} \text {. }
$$

Thus, for every data set, 33 chi-square values were computed. The results were as follows:

(1) With the exception of 2 or 3 of the 17 data sets, the last mentioned version (Equation 15) gave a worse fit than the break version.
(2) Introducing different $\phi_{0}$ values was about equivalent to introducing different $\alpha$ values. This result is somewhat disappointing because one cannot pinpoint the pertinent parameter. Such an outcome seems not to be unusual, however (see, e.g., Falmagne, Iverson, \& Marcovici, 1979, Appendix C). The choice of which parameter varies is, in any case, not crucial for the PC model, as such. Since a variation in $\phi_{0}$ yielded somewhat lower chi-squares on the average, this alternative was chosen.

(3) Using both different $\phi_{0}$ and different $\alpha$ values improved the fit only negligibly-compared with adding only one parameter-for all observers except L.P. $\left(\chi^{2}=4.0\right)$ and, for the short durations, J.B. $\left(\chi^{2}=11.3\right)$. In both cases, the corresponding $s$ values were extremely small, leading me to believe that the hypersurfaces on which the minima are searched are endowed with misleading "potholes."

(4) One might suspect that the search for the location of the break is, by adding a few parameters, just a way of capitalizing on chance. The data, however, obviate this objection. Typically, the chi-square values for the breaks adjacent to the optimal break location are roughly twice as large. This outcome strongly supports the concept of breaks in these psychophysical functions. One exception is $\mathrm{Ob}$ server J.B. (long durations), for whom all chi-square values scatter around 7 . Obviously, his psychophysical function does not show any break (within the experimental range), and the small improvement found when another $\phi_{0}$ parameter is added is just a capitalization on chance.

In view of the four points listed above, a break for all observers and different $\phi_{0}$ values on the two sides of the break were assumed. Table 4 gives the parameter and chi-square values for all observers, both with and without a break and with the location of the break. It is seen that the introduction of a break decreases the chi-square values dramatically.

The PC model with a break is statistically not very tractable. It is not clear whether the computed chisquare statistic really has a chi-square distribution, or, even if it has, with how many degrees of freedom. It seems, therefore, difficult to test whether the introduction of a break confers a statistically significant improvement. Tentatively, we will assume that the chi-square statistic for the PC model with break is chi-square distributed with between 6 and 5 degrees of freedom. The reason is that the addition of one $\phi_{0}$ value increases the number of parameters by one. The location of the break, on the other hand, cannot, and need not, be determined precisely-only 1 of 10 alternatives has to be chosen. Therefore, the location of the break ought not to increase the number of parameters by unity.

Thus, assuming 5 degrees of freedom, we find 11 acceptable fits $(p>.3)$ out of 17 possible; for 6 degrees of freedom, we find 12 acceptable fits. For both 5 and 6 degrees of freedom, we have to reject one fit 
Table 4

Parameter and Chi-Square Values for the Parallel-Clock Model for Each Subject in Allan's (1977) Experiments 1 and 2

\begin{tabular}{|c|c|c|c|c|c|c|c|c|c|c|}
\hline \multirow[b]{2}{*}{ Subject } & \multicolumn{4}{|c|}{ Without Break } & \multicolumn{6}{|c|}{ With Break } \\
\hline & $\phi_{0}{ }^{*}$ & $\beta$ & $\mathrm{s}$ & $x^{2}$ & $\begin{array}{c}\text { Break at } \\
\text { About* }\end{array}$ & $\phi_{0}^{*}$ & $\phi_{0}^{*}$ & $\beta$ & $\mathbf{s}$ & $x^{2}$ \\
\hline & & & & & Long D & tions & & & & \\
\hline $\begin{array}{l}\text { L.P. } \\
\text { S.S. } \\
\text { B.J. } \\
\text { K.F. } \\
\text { S.M. } \\
\text { J.B. } \\
\text { J.A. } \\
\text { L.S. }\end{array}$ & $\begin{array}{r}.480 \\
-.000 \\
.159 \\
.294 \\
.469 \\
.324 \\
.315 \\
.691\end{array}$ & $\begin{array}{r}.62 \\
1.10 \\
.82 \\
.68 \\
.78 \\
.75 \\
.76 \\
.47\end{array}$ & $\begin{array}{l}.164 \\
.233 \\
.136 \\
.189 \\
.435 \\
.110 \\
.134 \\
.284\end{array}$ & $\begin{array}{r}27.8 \\
4.9 \\
21.8 \\
17.8 \\
12.1 \\
7.7 \\
4.9 \\
8.3\end{array}$ & $\begin{array}{r}2.250 \\
.950 \\
2.350 \\
.950 \\
1.850 \\
1.050 \\
2.050 \\
1.850\end{array}$ & $\begin{array}{r}.448 \\
-.178 \\
.175 \\
-.136 \\
.284 \\
.404 \\
.352 \\
.496\end{array}$ & $\begin{array}{r}.656 \\
-.149 \\
.282 \\
-.061 \\
.510 \\
.391 \\
.394 \\
.736\end{array}$ & $\begin{array}{r}.48 \\
1.23 \\
.73 \\
.90 \\
.66 \\
.70 \\
.70 \\
.39\end{array}$ & $\begin{array}{l}.166 \\
.297 \\
.122 \\
.285 \\
.390 \\
.103 \\
.129 \\
.289\end{array}$ & $\begin{array}{r}11.2 \\
1.1 \\
11.2 \\
5.8 \\
4.3 \\
5.9 \\
2.6 \\
4.6\end{array}$ \\
\hline L.S. & \multicolumn{10}{|c|}{ Short Durations } \\
\hline $\begin{array}{l}\text { M.G. } \\
\text { L.S. } \\
\text { P.B. } \\
\text { S.A. } \\
\text { D.O. } \\
\text { D.E. } \\
\text { B.J. } \\
\text { J.B. } \\
\text { J.A. }\end{array}$ & $\begin{array}{l}.020 \\
.047 \\
.041 \\
.034 \\
.026 \\
.054 \\
.030 \\
.009 \\
.036\end{array}$ & $\begin{array}{l}.89 \\
.70 \\
.80 \\
.74 \\
.85 \\
.67 \\
.69 \\
.89 \\
.77\end{array}$ & $\begin{array}{l}.030 \\
.061 \\
.040 \\
.074 \\
.040 \\
.137 \\
.095 \\
.027 \\
.041\end{array}$ & $\begin{array}{r}12.5 \\
7.4 \\
27.8 \\
12.5 \\
16.5 \\
25.9 \\
7.9 \\
34.6 \\
9.5\end{array}$ & $\begin{array}{l}.185 \\
.275 \\
.245 \\
.135 \\
.135 \\
.165 \\
.150 \\
.165 \\
.185\end{array}$ & $\begin{array}{r}.015 \\
.054 \\
.046 \\
.048 \\
.039 \\
-.030 \\
.040 \\
-.001 \\
.027\end{array}$ & $\begin{array}{l}.024 \\
.044 \\
.030 \\
.041 \\
.034 \\
.052 \\
.033 \\
.009 \\
.040\end{array}$ & $\begin{array}{l}.84 \\
.73 \\
.91 \\
.71 \\
.81 \\
.51 \\
.69 \\
.85 \\
.71\end{array}$ & $\begin{array}{l}.032 \\
.056 \\
.032 \\
.071 \\
.039 \\
.164 \\
.087 \\
.029 \\
.046\end{array}$ & $\begin{array}{r}4.8 \\
2.5 \\
4.8 \\
7.4 \\
6.7 \\
9.8 \\
2.5 \\
25.4 \\
5.1\end{array}$ \\
\hline
\end{tabular}

Note-The first $\phi_{0}$ value on the right-hand side of the table refers to the portion of the psychophysical function above the break; the second refers to the one below the break. *In seconds.

at the .001 level of significance, and for 5 degrees of freedom, two more at the .05 level.

In order to investigate the overall fit, the KolmogorovSmirnov test is used. Here, it tests, in addition to the model, implicitly the distribution of the test variable, too; that is, it tests to determine whether the distribution of a sample for $n=17$ agrees with the corresponding theoretical distribution. Assuming a chisquare distribution with 6 and 5 degrees of freedom, the Kolmogorov-Smirnov statistics are .090 and .193 , respectively, far lower values than that which corresponds to the .3 point of the Kolmogorov-Smirnov distribution for $n=17$. This is extremely satisfactory, although it should, of course, be remembered that the Kolmogorov-Smirnov test has low power, particularly for such a low $n$.

Figure 2 shows the fit for all data sets in terms of predicted and observed proportions of "first longer" responses. Remember that the computations, minimizing chi-square, were not optimal for the data as represented in this figure. The largest chi-square (for J.B., short durations) may at least in part be due to the extreme frequencies, close to $5 \%$ and $90 \%$. These proportions, with few responses in one of the two categories, are relatively uncertain and influence the chi-square heavily. Note also that D.E. shows an inconsistency in the data: he had a higher frequency of "first longer" judgments for the pair .13, .16 sec than for the pair $.16, .16 \mathrm{sec}$.

One could hypothesize that the (implicit) assumption of a constant comparatal dispersion is incorrect. Table 4 shows clearly that the $s$ values for the longer durations are higher than those for the shorter. This observation may indicate that, within an experiment also, $s$ grows with the stimulus values. An attempt to compute s "backwards," that is, from the other parameters and the empirical proportions, did not show any clear trend. The scatter was large for most of the observers, and both increasing and decreasing trends could be seen, as well as nearly constant $s$ values.

It is to be expected-and it is borne out-that the $\phi_{0}$ values are greater for the longer durations. A more interesting question is whether the exponent depends on the average duration of the stimuli. A t test between the data for long and short durations' yields a $t$ value of .29. Accordingly, there seems to be no difference in the exponent for the two groups. The mean exponent for the 17 observers is .74. This is somewhat lower than the average of .9 found by Eisler (1976). It is still astonishingly close, taking the difference between scaling and discrimination experiments into account.

\section{CONCLUDING REMARKS}

It is found that the parallel-clock model accounts very well for discrimination data, as proposed earlier (Eisler, 1975). It may be of interest to note that the term "time-order error," a problem that induced Allan to carry out the experiments on which the present study is based, has not been used. Once the process of duration discrimination is known, the timeorder error is seen to be a simple consequence of it. 

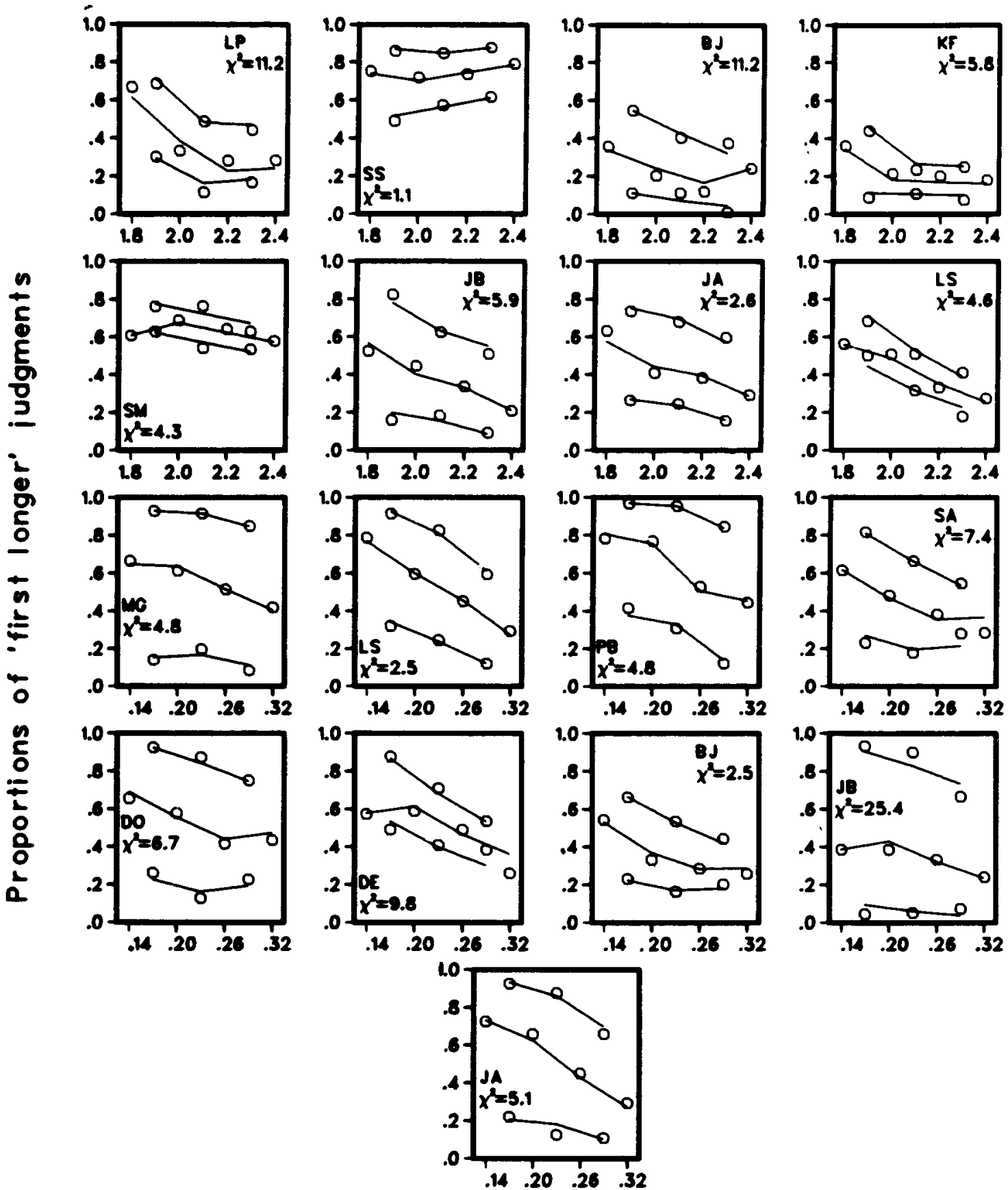

Total duration in sec

Figure 2. Proportion of "first longer" judgments as a function of the total physical duration for all 17 data sets. The lines connect predicted proportions for the following three cases: (a) first stimulus longer than second, (b) both stimuli equal, (c) first stimulus shorter than second. The symbols show the empirical values. 


\section{REFERENCE NOTE}

1. Jamieson, D. G. Personal communication, August 1978.

\section{REFERENCES}

Allan, L. G. The time-order error in judgments of duration. Canadian Journal of Psychology, 1977, 31, 24-31.

Chandler, J. STEPIT - Finds local minima of a smooth function of several parameters. Behavioral Science, 1969, 14, 81-82. (CPA 312)

EisLer, H. Subjective duration and psychophysics. Psychological Review, 1975, 82, 429-450.

Eisle R, H. Experiments on subjective duration 1868-1975: A collection of power function exponents. Psychological Bulletin, $1976,83,1154-1171$.

Falmagne, J. C., Iverson, G., \& Marcovici, S. Binaural "loudness" summation: Probabilistic theory and data. Psychological Review, 1979, 86, 25-43.

Guilford, J. P. Psychometric methods. New York: McGraw-Hill, 1954.

Hellström, $\AA$. Time errors are perceptual. An experimental investigation of duration and a quantitative successivecomparison model. Psychological Research, 1977, 39, 345-388.

Jamieson, D. G. Two presentation order effects. Canadian Journal of Psychology, 1977, 31, 184-194.

Kristofferson, A. B. A real-time criterion theory of duration discrimination. Perception \& Psychophysics, 1977, 21, 105-117.

Luce, R. D. Individual choice behavior. New York: Wiley, 1959.

Luce, R. D., \& Galanter, E. Discrimination. In R. D. Luce, R. R. Bush, \& E. Galanter (Eds.), Handbook of mathematical psychology (Vol. 1). New York: Wiley, 1963.

\section{NOTES}

1. The interstimulus interval might pose some problems. It is not taken into account in the present context; it might be "omitted" by the observer by, for example, "switching off" the register during its duration, or it might be absorbed by the subjective zero $\phi_{0}$ (see below).

2. Far from all of the authors of the 111 studies collected were aware that they were carrying out scaling experiments. However, the PC model could be applied to their data and would produce scales of duration.

3. In her paper, Allan (1977) tabled only proportions of "first longer" responses and mentioned the approximate number of presentations of each pair. However, she kindly made her raw data available to me.

4. The distribution function of the difference of two triangular distributions is:

$$
\begin{array}{ll}
\frac{1}{24}(\zeta+2)^{4} & \text { for }-2 \leqslant \zeta \leqslant-1 \\
\frac{1}{24}(\zeta+2)^{4}-\frac{1}{6}(\zeta+1)^{4} & \text { for }-1 \leqslant \zeta \leqslant 0 \\
\frac{1}{24}(\zeta+2)^{4}-\frac{1}{6}(\zeta+1)^{4}+\frac{1}{4} \zeta & \text { for } 0 \leqslant \zeta \leqslant 1 \\
\frac{1}{24}(\zeta+2)^{4}-\frac{1}{6}(\zeta+1)^{4}+\frac{1}{4} \zeta^{4}-\frac{1}{6}(\zeta-1)^{4} & \text { for } 1 \leqslant \zeta \leqslant 2 .
\end{array}
$$

5. The observant reader may wonder why the chi-square values given by Jamieson (1977) are roughly half those in Table 3. The reason is not so much that Jamieson calculated the frequencies of "first longer" responses from the proportions given by Allan (1977), while I used the exact raw data; rather, the real reason is that Jamieson computed the chi-square values only for the "first longer" response frequencies, omitting the "second longer" ones. Thus, he calculated

$$
\chi^{2}=\sum_{i=1}^{10} \frac{\left(O_{1 i}-P_{1 i}\right)^{2}}{P_{1 i}}
$$

instead of

$$
\chi^{2}=\sum_{\mathrm{i}=1}^{10}\left[\frac{\left(\mathrm{O}_{1 \mathrm{i}}-\mathrm{P}_{1 \mathrm{i}}\right)^{2}}{\mathrm{P}_{1 \mathrm{i}}}+\frac{\left(\mathrm{O}_{2 \mathrm{i}}-\mathrm{P}_{2 \mathrm{i}}\right)^{2}}{\mathrm{P}_{2 \mathrm{i}}}\right] .
$$

Otherwise his calculations were correct: I repeated his calculations with the values given in his Table 1 , minimizing in accordance with the first formula above, and obtained the same results, in spite of our using different computer programs (Jamieson, Note 1).

6. At the moment, I do not have any convincing theory for the breaks in the psychophysical functions. It seems to me to be analogous to the change in scale of a voltmeter from $100 \mathrm{~V}$, say, to $10 \mathrm{~V}$, to maximize reading precision.

7. Considering the low values of $t$, the fact that $t$ has been computed for independent samples, though four of the observers appear in both groups, may be neglected. However, for those who prefer loss of power to tampering with statistical prescriptions, I also calculated $t$ for data deriving from Allan's Experiment 1 only (seven observers for the long and six for the short durations) and obtained $t=.18$. For two of the four observers who participated in both experiments, the exponents agreed closely, whereas there were sizable differences for the other two.

(Received for publication June 30, 1980; revision accepted November 26,1980 .) 